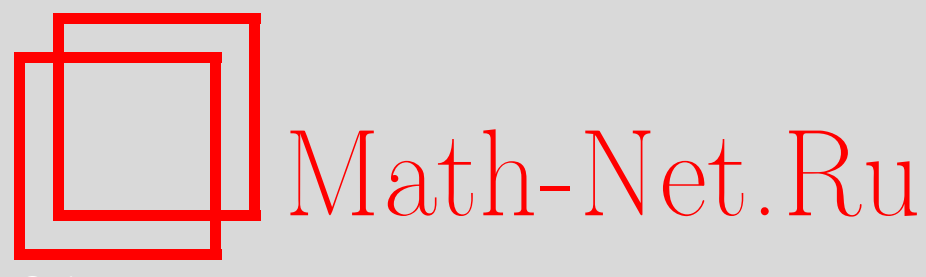

М. Э. Муминов, Н. М. Алиев, О дискретном спектре некомпактного возмущения трехчастичного оператора Шредингера на решетке, ТМФ, 2015, том 182, номер 3, 435-452

DOI: https://doi.org/10.4213/tmf8764

Использование Общероссийского математического портала Math-Net.Ru подразумевает, что вы прочитали и согласны с пользовательским соглашением http: //www . mathnet.ru/rus/agreement

Параметры загрузки:

IP : 54.92 .164 .108

26 апреля 2023 г., 16:24:51

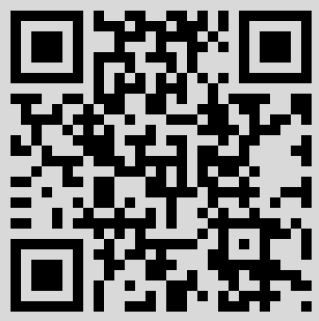




\title{
О ДИСКРЕТНОМ СПЕКТРЕ НЕКОМПАКТНОГО ВОЗМУЩЕНИЯ ТРЕХЧАСТИЧНОГО ОПЕРАТОРА ШРЕДИНГЕРА НА РЕШЕТКЕ
}

\begin{abstract}
Рассмотрена система трех произвольных квантовых частиц на трехмерной решетке, взаимодействующих с помощью парных контактных потенциалов притяжения и потенциалов притяжения частиц в ближайших соседних узлах. Доказана бесконечность числа собственных значений гамильтониана соответствующей системы трех частиц. Указаны различные типы потенциалов притяжения, для которых собственные значения могут лежать левее существенного спектра, в лакуне существенного спектра, а также в существенном спектре рассматриваемого оператора.
\end{abstract}

Ключевые слова: трехчастичная система на решетке, оператор Шредингера, асимптотика числа собственных значений, бесконечность числа собственных значений в лакуне существенного спектра, бесконечность числа собственных значений в существенном спектре.

DOI: $10.4213 / \operatorname{tmf} 8764$

\section{1. ВВЕДЕНИЕ}

Явление существования бесконечного числа собственных значений, накапливающихся к левому краю существенного спектра оператора Шредингера (эффект Ефимова [1]), изучалось во многих работах (см., например, [2], [3]). Бесконечность числа собственных значений трехчастичного оператора Шредингера и асимптотика для их числа получены первоначально в статье [4], затем в работе [5], а также, в случае оператора на решетке, в работах [6]-[8]. В этих работах для получения указанной асимптотики построен компактный оператор $A(z)$, заданный для $z$, лежащих левее существенного спектра, такой, что $N(z)=n(1, A(z))$, где $N(z)$ и $n(\lambda, A)$ - число собственных значений оператора Шредингера и оператора $A(z)$, лежащих соответственно левее $z$ и правее $\lambda$.

${ }^{*}$ Faculty of Science, Universiti Teknologi Malaysia, Johor Bahru, Malaysia. E-mail: mmuminov@mail.ru

* Механико-математический факультет, Самаркандский государственный университет, Самарканд, Узбекистан. E-mail: nialiyev@mail.ru 
Заметим, что в работах [6]-[9] бесконечное число собственных значений появляется ниже существенного спектра в случае, когда два из трех двухчастичных подгамильтонианов имеют виртуальные уровни в нуле и нуль является регулярной точкой для третьего гамильтониана или когда все двухчастичные подгамильтонианы имеют виртуальные уровни в нуле.

Непрерывный спектр трехчастичного (непрерывного) оператора Шредингера совпадает с полуосью $\left[-\kappa^{2}, \infty\right), \kappa \geqslant 0[10]$. В отличие от существенного спектра трехчастичного непрерывного оператора Шредингера существенный спектр $\sigma_{\mathrm{ess}}(H(K))$ трехчастичного дискретного оператора Шредингера $H(K)$ может содержать лакуны, т. е. существует интервал $(a, b)$,

$$
\inf \sigma_{\mathrm{ess}}(H(K)) \leqslant a<b \leqslant \sup \sigma_{\mathrm{ess}}(H(K)),
$$

такой, что $(a, b) \cap \sigma_{\text {ess }}(H(K))=\varnothing$.

В работе [11] рассматривается система трех произвольных квантовых частиц на трехмерной решетке, взаимодействующих с помощью парных контактных потенциалов притяжения. Рассмотрен случай, когда два двухчастичных подгамильтониана имеют виртуальные уровни в нуле и нуль является регулярной точкой для третьего подгамильтониана. Найдено условие появления лакуны существенного спектра, и при нулевом значении полного квазиимпульса $K$ доказано существование бесконечного числа собственных значений оператора $H(\mathbf{0})$ в лакуне существенного спектра. В работе [12] для системы трех произвольных квантовых частиц на трехмерной решетке, взаимодействующих с помощью короткодействующих потенциалов притяжения, построен самосопряженный компактный оператор $\mathbf{T}(z)$, определенный при $z$, лежащих левее существенного спектра оператора $H(K)$ (соответствующего трехчастичной системе) и в лакуне существенного спектра, если такая лакуна существует. Получена формула для числа собственных значений, лежащих в произвольном интервале вне существенного спектра этого оператора, и, в частности, доказано равенство $N(z)=n(1, \mathbf{T}(z))$ при $z<\inf \sigma_{\text {ess }}(H(K))$, а также найдено достаточное условие конечности дискретного спектра.

В настоящей работе рассматриваются возмущения системы трех произвольных квантовых частиц на трехмерной решетке, взаимодействующих с помощью парных контактных потенциалов притяжения, где в качестве возмущений берутся потенциалы притяжения частиц, взаимодействующих только в ближайших соседних узлах. Изучаются спектральные свойства соответствующего трехчастичного оператора Шредингера на решетке

$$
\bar{H}^{\lambda}(K)=H(K)-\bar{V}^{\lambda}, \quad K \in \mathbb{T}^{3}=(-\pi, \pi]^{3}, \quad \lambda=\left(\lambda_{1}, \lambda_{2}, \lambda_{3}\right),
$$

при нулевом значении квазиимпульса $K=\mathbf{0}$. Сначала доказывается бесконечность числа точек дискретного спектра невозмущенного оператора $\bar{H}^{0}(\mathbf{0}):=H(\mathbf{0})$, накапливающихся в нуле, т. е. на левом краю существенного спектра $\sigma_{\mathrm{ess}}(H(\mathbf{0}))$. Затем показывается, что пространство $\mathcal{H}$ собственных функций оператора $H(\mathbf{0})$, соответствующих собственным значениям, лежащим в дискретном спектре, является подпространством ядра оператора возмущения $\bar{V}^{\lambda}$.

Отметим, что существенный спектр $\sigma_{\text {ess }}\left(\bar{H}^{\lambda}(K)\right)$ оператора $\bar{H}^{\lambda}(K)$ представляет собой объединение спектров канальных операторов $\bar{H}_{\alpha}^{\lambda}(K), \alpha=1,2,3$. Мы находим 
(см. доказательство леммы 4) такие значения $\lambda_{\alpha}^{0}>0$, что при положительных $\lambda_{\alpha}$, меньших этих значений, спектр оператора $\bar{H}_{\alpha}^{\lambda}(\mathbf{0})$ не меняется и совпадает со спектром невозмущенного канального оператора $\bar{H}_{\alpha}^{0}(\mathbf{0})$, а при некоторых положительных значениях $\lambda_{\alpha}$, больших этих значений, в спектре оператора $\bar{H}_{\alpha}^{\lambda}(\mathbf{0})$ появляется некоторый отрезок, который находится слева от существенного спектра $\sigma_{\mathrm{ess}}(H(\mathbf{0}))$ и удаляется от него по мере увеличения $\lambda_{\alpha}$. При этом наблюдается интересная картина: при некоторых значениях $\lambda$ бесконечное число собственных значений оператора $\bar{H}^{\lambda}(\mathbf{0})$ появляется в существенном спектре (или на левом краю существенного спектра), а при больших значениях $|\lambda|$ найденные собственные значения могут находиться в лакуне существенного спектра оператора Шредингера $\bar{H}^{\lambda}(\mathbf{0})$.

\section{2. ОПИСАНИЕ ТРЕХЧАСТИЧНОГО ОПЕРАТОРА И ФОРМУЛИРОВКА ОСНОВНЫХ РЕЗУЛЬТАТОВ}

Пусть $\mathbb{Z}^{3}$ - трехмерная целочисленная решетка и $\ell_{2}\left(\left(\mathbb{Z}^{3}\right)^{3}\right)$ - гильбертово пространство квадратично-суммируемых функций, определенных на $\left(\mathbb{Z}^{3}\right)^{3}$.

Рассмотрим свободный гамильтониан $\widehat{H}_{0}$ системы трех произвольных квантовых частиц на решетке $\mathbb{Z}^{3}$, определенный как ограниченный самосопряженный оператор в гильбертовом пространстве $\ell_{2}\left(\left(\mathbb{Z}^{3}\right)^{3}\right)$, действующий по правилу

$$
\begin{aligned}
& \left(\widehat{H}_{0} \hat{\psi}\right)\left(n_{1}, n_{2}, n_{3}\right)= \\
& \quad=\sum_{s \in \mathbb{Z}^{3}}\left[\hat{\varepsilon}_{1}(s) \hat{\psi}\left(n_{1}+s, n_{2}, n_{3}\right)+\hat{\varepsilon}_{2}(s) \hat{\psi}\left(n_{1}, n_{2}+s, n_{3}\right)+\hat{\varepsilon}_{3}(s) \hat{\psi}\left(n_{1}, n_{2}, n_{3}+s\right)\right] .
\end{aligned}
$$

Здесь $\hat{\varepsilon}_{\alpha}(s), \alpha=1,2,3$, - вещественнозначные четные функции, описывающие перенос частицы с узла на соседний узел:

$$
\hat{\varepsilon}_{\alpha}(s)=\left\{\begin{array}{cl}
\frac{3}{m_{\alpha}} & \text { при } \quad s=0, \\
-\frac{1}{2 m_{\alpha}} & \text { при } s= \pm 2 e_{j}, j=1,2,3, \\
0 & \text { в остальных случаях, }
\end{array}\right.
$$

где $m_{\alpha}>0$ - масса частицы с номером $\alpha=1,2,3$ и $e_{j}, j=1,2,3,-$ единичные орты.

Заметим, что свободный гамильтониан $\widehat{H}_{0}$ системы трех произвольных частиц в пространстве $\ell_{2}\left(\left(\mathbb{Z}^{3}\right)^{3}\right)$ можно представить в виде

$$
\widehat{H}_{0}=\frac{1}{2 m_{1}} P_{2}(\Delta) \times E \times E+\frac{1}{2 m_{2}} E \times P_{2}(\Delta) \times E+\frac{1}{2 m_{3}} E \times E \times P_{2}(\Delta),
$$

где $E$ - единичный оператор в $\ell_{2}\left(\mathbb{Z}^{3}\right), \Delta$ - решеточный лапласиан,

$$
(\Delta \hat{\psi})(x)=\sum_{|s|=1}[\hat{\psi}(x)-\hat{\psi}(x+s)], \quad \hat{\psi} \in \ell_{2}\left(\mathbb{Z}^{3}\right),
$$

и $P_{2}(\Delta)=\Delta^{2}-4 \Delta$.

Трехчастичный гамильтониан $\widehat{H}$ системы трех квантовых частиц с парными контактными потенциалами определяется как ограниченное возмущение свободного гамильтониана $\widehat{H}_{0}$ :

$$
\widehat{H}=\widehat{H}_{0}-\mu_{1} \widehat{V}_{1}-\mu_{1} \widehat{V}_{2}-\mu_{3} \widehat{V}_{3}
$$


где

$$
\left(\widehat{V}_{\alpha} \psi\right)\left(n_{1}, n_{2}, n_{3}\right)=\delta_{n_{\beta} n_{\gamma}} \psi\left(n_{1}, n_{2}, n_{3}\right), \quad \alpha \neq \beta \neq \gamma \text {. }
$$

Здесь $\mu_{\alpha}>0$ - энергия взаимодействия двух частиц, $\delta_{n m}-$ символ Кронекера.

Пусть $\lambda_{\alpha}>0$ для $\alpha=1,2,3$ и $\widetilde{V}^{\lambda}=\lambda_{1} \widetilde{V}_{1}+\lambda_{2} \widetilde{V}_{2}+\lambda_{3} \widetilde{V}_{3}-$ сумма потенциалов притяжения двух частиц, взаимодействующих только в ближайших соседних узлах:

$$
\left(\widetilde{V}_{\alpha} \psi\right)\left(n_{1}, n_{2}, n_{3}\right)=\tilde{v}\left(n_{\beta}-n_{\gamma}\right) \psi\left(n_{1}, n_{2}, n_{3}\right), \quad \alpha \neq \beta \neq \gamma,
$$

где

$$
\tilde{v}(s)=\left\{\begin{array}{cl}
4 \pi^{3} & \text { при } \quad s= \pm e_{j}, j=1,2,3 \\
0 & \text { в остальных случаях. }
\end{array}\right.
$$

В дальнейшем мы будем рассматривать возмущения $\widehat{H}-\widetilde{V}^{\lambda}$ трехчастичного гамильтониана $\widehat{H}$ потенциалом $-\widetilde{V}^{\lambda}$.

Пусть $L_{2}\left(\left(\mathbb{T}^{3}\right)^{n}\right)$ - гильбертово пространство квадратично-интегрируемых функций, определенных на $\left(\mathbb{T}^{3}\right)^{n}$, где $\mathbb{T}=(-\pi, \pi], n=1,2,3$. При использовании преобразования Фурье и разложения в прямой операторный интеграл [12], [13] изучение спектральных свойств оператора $\widehat{H}-\widetilde{V}^{\lambda}$ сводится к исследованию спектральных свойств семейства самосопряженных ограниченных операторов $\bar{H}^{\lambda}(K), K \in \mathbb{T}^{3}$, действующих в гильбертовом пространстве $L_{2}\left(\left(\mathbb{T}^{3}\right)^{2}\right)$ по формуле

$$
\bar{H}^{\lambda}(K)=H(K)-\bar{V}^{\lambda},
$$

где

$$
H(K)=H_{0}(K)-V, \quad V=\mu_{1} V_{1}+\mu_{2} V_{2}+\mu_{3} V_{3}, \quad \bar{V}^{\lambda}=\lambda_{1} \bar{V}_{1}+\lambda_{2} \bar{V}_{2}+\lambda_{3} \bar{V}_{3} .
$$

Здесь $H_{0}(K)$ - оператор умножения на функцию

$$
E_{K}(p, q)=\frac{1}{m_{1}} \varepsilon(p)+\frac{1}{m_{2}} \varepsilon(q)+\frac{1}{m_{3}} \varepsilon(K-p-q), \quad \varepsilon(p)=\sum_{i=1}^{3}\left(1-\cos 2 p_{i}\right),
$$

а операторы $V_{\alpha}$ и $\bar{V}_{\alpha}, \alpha=1,2,3$, заданы следующим образом:

$$
\begin{gathered}
\left(V_{1} f\right)(p, q)=\frac{1}{(2 \pi)^{3}} \int_{\mathbb{T}^{3}} f(p, s) d s, \quad\left(V_{2} f\right)(p, q)=\frac{1}{(2 \pi)^{3}} \int_{\mathbb{T}^{3}} f(s, q) d s, \\
\left(V_{3} f\right)(p, q)=\frac{1}{(2 \pi)^{3}} \int_{\mathbb{T}^{3}} f(s, p+q-s) d s \\
\left(\bar{V}_{1} f\right)(p, q)=\int_{\mathbb{T}^{3}} v(s-p) f(s, p) d s, \quad\left(\bar{V}_{2} f\right)(p, q)=\int_{\mathbb{T}^{3}} v(s-q) f(s, q) d s, \\
\left(\bar{V}_{3} f\right)(p, q)=\int_{\mathbb{T}^{3}} v(s-p) f(s, p+q-s) d s, \quad v(p)=\sum_{i=1}^{3} \cos p_{i} .
\end{gathered}
$$

Здесь $f \in L_{2}\left(\left(\mathbb{T}^{3}\right)^{2}\right)$.

Введем оператор Шредингера $\bar{h}_{\alpha}(k), k \in \mathbb{T}^{3}$, соответствующий гамильтониану системы двух частиц на решетке, который действует в пространстве $L_{2}\left(\mathbb{T}^{3}\right)$ по формуле

$$
\bar{h}_{\alpha}(k)=h_{\alpha}(k)-\lambda_{\alpha} \overline{\mathbf{v}}, \quad \alpha=1,2,3,
$$


где $h_{\alpha}(k)=h_{0}^{\alpha}(k)-\mu_{\alpha} \mathbf{v}$ и $h_{0}^{\alpha}(k)$ - оператор умножения на функцию

$$
\mathcal{E}_{k}^{(\alpha)}(p)=\frac{1}{m_{\beta}} \varepsilon(p)+\frac{1}{m_{\gamma}} \varepsilon(k-p),
$$

а $\overline{\mathbf{v}}$ и $\mathbf{v}$ - интегральные операторы с ядрами $v(p-s)$ и $t(p, s)=1 /(2 \pi)^{3}$ соответственHO.

Пусть $C\left(\mathbb{T}^{3}\right)$ - банахово пространство непрерывных (периодических) функций на $\mathbb{T}^{3}$ и $G_{\alpha}$ - интегральный оператор (Бирмана-Швингера) с ядром

$$
G_{\alpha}(p, q)=\frac{1}{8 \pi^{3}} \frac{1}{\mathcal{E}_{\mathbf{0}}^{(\alpha)}(q)}, \quad p, q \in \mathbb{T}^{3} .
$$

ОПРЕДЕЛЕНИЕ 1. Если единица является собственным значением оператора $G_{\alpha}$ и соответствующая собственная функция $\psi$ удовлетворяет условию

$$
\frac{\psi(\cdot)}{\mathcal{E}_{\mathbf{0}}^{(\alpha)}(\cdot)} \in L_{1}\left(\mathbb{T}^{3}\right) \backslash L_{2}\left(\mathbb{T}^{3}\right),
$$

то говорят, что оператор $h_{\alpha}(\mathbf{0})$ имеет виртуальный уровенъ в нуле (на левом краю существенного спектра).

Простое вычисление показывает, что оператор $h_{\alpha}(\mathbf{0})$ имеет виртуальный уровень в нуле тогда и только тогда, когда $\mu_{\alpha}=\mu_{\alpha}^{0}$, где

$$
\mu_{\alpha}^{0}=8 \pi^{3} \frac{m_{\beta}+m_{\gamma}}{m_{\beta} m_{\gamma}}\left(\int_{\mathbb{T}^{3}} \frac{d s}{\varepsilon(s)}\right)^{-1} .
$$

Оператор $\bar{H}_{\alpha}^{\lambda}(K)=H_{0}(K)-\mu_{\alpha} V_{\alpha}-\lambda_{\alpha} \bar{V}_{\alpha}$ называется канальным оператором и представляется в виде прямого интеграла [12], [13]:

$$
\bar{H}_{\alpha}^{\lambda}(K)=\int_{\mathbb{T}^{3}} \bigoplus\left[\hat{h}_{\alpha}(K-p)+\frac{\varepsilon(p)}{m_{\alpha}}\right] d p,
$$

где оператор $\hat{h}_{\alpha}(p)$ унитарно эквивалентен оператору $\bar{h}_{\alpha}(p)$. Из этого представления и в силу теоремы о спектре тензорных произведений операторов имеем

$$
\sigma\left(\bar{H}_{\alpha}^{\lambda}(K)\right)=\bigcup_{p \in \mathbb{T}^{3}}\left[\sigma\left(\bar{h}_{\alpha}(K-p)+\frac{\varepsilon(p)}{m_{\alpha}}\right)\right] .
$$

Из теоремы Вейля о существенном спектре [14] следует, что существенный спектр $\sigma_{\text {ess }}\left(\bar{h}_{\alpha}(k)\right)$ оператора $\bar{h}_{\alpha}(k)$ не меняется при компактом возмущении $\mu_{\alpha} \mathbf{v}_{\alpha}+\lambda_{\alpha} \overline{\mathbf{v}}$ и совпадает со спектром невозмущенного оператора $h_{0}^{\alpha}(k)$. При этом $\sigma_{\text {ess }}\left(\bar{h}_{\alpha}(k)\right)$ совпадает с областью значений функции $\mathcal{E}_{k}^{(\alpha)}(\cdot)$, т. е.

$$
\sigma_{\mathrm{ess}}\left(\bar{h}_{\alpha}(k)\right)=\sigma\left(h_{0}^{\alpha}(k)\right)=\left[m^{(\alpha)}(k), M^{(\alpha)}(k)\right],
$$

где

$$
m^{(\alpha)}(k)=\min _{p} \mathcal{E}_{k}^{(\alpha)}(p), \quad M^{(\alpha)}(k)=\max _{p} \mathcal{E}_{k}^{(\alpha)}(p)
$$


Поэтому из (2) вытекает, что

$$
\begin{aligned}
& \sigma\left(\bar{H}_{\alpha}^{\lambda}(K)\right)= \\
& =\bigcup_{p \in \mathbb{T}^{3}}\left\{\left[m^{(\alpha)}(K-p)+\frac{\varepsilon(p)}{m_{\alpha}}, M^{(\alpha)}(K-p)+\frac{\varepsilon(p)}{m_{\alpha}}\right] \bigcup \sigma_{\operatorname{disc}}\left(\bar{h}_{\alpha}(K-p)+\frac{\varepsilon(p)}{m_{\alpha}}\right)\right\}
\end{aligned}
$$

или, эквивалентно,

$$
\sigma\left(\bar{H}_{\alpha}^{\lambda}(K)\right)=\left\{\bigcup_{p \in \mathbb{T}^{3}} \sigma_{\operatorname{disc}}\left(\bar{h}_{\alpha}(K-p)+\frac{\varepsilon(p)}{m_{\alpha}}\right)\right\} \bigcup\left[m_{K}, M_{K}\right]
$$

где

$$
m_{K}=\min _{p, q} E_{K}(p, q), \quad M_{K}=\max _{p, q} E_{K}(p, q) .
$$

Следующая лемма является частным случаем теоремы 3.2 из работы [13].

Лемма 1. Для любого $K \in \mathbb{T}^{3}$ существенный спектр $\sigma_{\text {ess }}\left(\bar{H}^{\lambda}(K)\right)$ оператора $\bar{H}^{\lambda}(K)$ представляет собой объединение спектров канальных операторов $\bar{H}_{\alpha}^{\lambda}(K)$ :

$$
\sigma_{e s s}\left(\bar{H}^{\lambda}(K)\right)=\sigma\left(\bar{H}_{1}^{\lambda}(K)\right) \cup \sigma\left(\bar{H}_{2}^{\lambda}(K)\right) \cup \sigma\left(\bar{H}_{3}^{\lambda}(K)\right) .
$$

Теперь сформулируем основные результаты настоящей работы.

Лемма 2. Пусть $\mu_{\alpha} \leqslant \mu_{\alpha}^{0}$ для $\alpha=1,2,3$. Тогда

$$
\sigma_{e s s}(H(\mathbf{0}))=\sigma_{e s s}\left(H_{0}(\mathbf{0})\right) .
$$

При этом $\inf \sigma_{\text {ess }}(H(\mathbf{0}))=0$ nри всех $\mu_{\alpha} \leqslant \mu_{\alpha}^{0}, \alpha=1,2,3$.

Данная лемма следует из спектральных свойств оператора $h_{\alpha}(k)$ и из равенства (3) при $\lambda_{\alpha}=0$ (см. лемму 7 из работы [11]).

Пусть $N(z)$ - число собственных значений оператора $H(\mathbf{0})$ (с учетом кратности), лежащих левее $z<0$. Следующая теорема утверждает существование бесконечного дискретного спектра оператора $H(\mathbf{0})$ и описывает асимптотику для $N(z)$ при $z \rightarrow-0$.

Tеорема 1. Пусть $\mu_{\alpha}=\mu_{\alpha}^{0}, \mu_{\beta}=\mu_{\beta}^{0} u \mu_{\gamma} \leqslant \mu_{\gamma}^{0}$ при $\alpha \neq \beta \neq \gamma$. Тогда сущеcmвуеm

$$
\lim _{z \rightarrow-0}\left(-\frac{1}{\ln (-z)} N(z)\right)=\mathcal{U}_{0}, \quad \mathcal{U}_{0}>0 .
$$

ЗАмЕчАниЕ 1. Положительное число $\mathcal{U}_{0}$ есть число, найденное в работах [4], [8], которое зависит от числа виртуальных уровней двухчастичных подгамильтонианов и от отношений масс частиц. В работе [8] функция, определяющая оператор умножения, имеет вид $E_{\mathbf{0}}(p / 2, q / 2)$, и она обладает единственной невырожденной точкой минимума. Отметим, что функция $E_{\mathbf{0}}(\cdot, \cdot)$ имеет 64 точки минимума. Теорема 1 показывает, что асимптотика для $N(z)$ при $z \rightarrow-0$ та же, что в работе [4], и она не зависит от числа точек минимума функции $E_{\mathbf{0}}(\cdot, \cdot)$.

ЛЕмма 3. Для любого $\lambda=\left(\lambda_{1}, \lambda_{2}, \lambda_{3}\right)$ каждое собственное значение $z$ оператора $H(\mathbf{0})$, лежащее левее его существенного спектра, является собственным значением оператора $\bar{H}^{\lambda}(\mathbf{0})$ с кратностью, не менъшей чем его кратность как собственного значения оператора $H(\mathbf{0})$. 
В следующей лемме описано местоположение существенного спектра оператоpa $\bar{H}^{\lambda}(\mathbf{0})$. Найдено условие, при котором существенный спектр оператора $\bar{H}^{\lambda}(\mathbf{0})$ содержит точку 0 (левый край спектра свободного гамильтониана) как внутреннюю точку, а также найдено условие появления лакуны.

В дальнейшем будем предполагать, что $\mu_{\alpha} \leqslant \mu_{\alpha}^{0}$ для всех $\alpha=1,2,3$.

Лемма 4. Справедливы следующие утверждения.

1. Существует $\lambda^{0}>0$ mакое, что для всех $\lambda=\left(\lambda_{1}, \lambda_{2}, \lambda_{3}\right)$ с $\lambda_{1,2,3} \leqslant \lambda^{0}$ uмеет место равенство

$$
\sigma\left(\bar{H}^{\lambda}(\mathbf{0})\right)=\sigma_{\text {ess }}\left(H_{0}(\mathbf{0})\right) .
$$

2. Существуют $\bar{\lambda}=\left(\bar{\lambda}_{1}, \bar{\lambda}_{2}, \bar{\lambda}_{3}\right)$ с $\bar{\lambda}_{1,2,3}>0$ и число $\bar{\rho}=\bar{\rho}(\bar{\lambda})>0$ такие, что имеет место включение

$$
[-\bar{\rho}, 0] \subset \sigma_{\mathrm{ess}}\left(\bar{H}^{\lambda}(\mathbf{0})\right) \quad \text { npu } \quad \lambda=\bar{\lambda}
$$

3. Существуют числа $\lambda^{*}>0$ и $\rho=\rho\left(\lambda^{*}\right)>0$ такие, что при всех $\lambda=\left(\lambda_{1}, \lambda_{2}, \lambda_{3}\right)$ $c \lambda_{1,2,3} \geqslant \lambda^{*}$

$$
\sigma_{\mathrm{ess}}\left(\bar{H}^{\lambda}(\mathbf{0})\right) \cap(-\rho, 0)=\varnothing \quad u \quad \sigma_{\mathrm{ess}}\left(\bar{H}^{\lambda}(\mathbf{0})\right) \cap(-\infty,-\rho) \neq \varnothing,
$$

точнее, существенный спектр $\sigma_{\mathrm{ess}}\left(\bar{H}^{\lambda}(\mathbf{0})\right)$ содержит лакуну $(-\rho, 0)$.

Из лемм 3, 4 и теоремы 1 вытекает следующая

ТЕОрема 2. Пусть $\mu_{\alpha}=\mu_{\alpha}^{0}, \mu_{\beta}=\mu_{\beta}^{0} u \mu_{\gamma} \leqslant \mu_{\gamma}^{0}$ для $\alpha \neq \beta \neq \gamma$. Тогда справедливы следующие утверждения.

1. Если $\lambda=\left(\lambda_{1}, \lambda_{2}, \lambda_{3}\right)$ таково, что $\lambda_{1,2,3} \leqslant \lambda^{0}$, mo оператор $\bar{H}^{\lambda}(\mathbf{0})$ uмеет бесконечное число собственных значений, лежащих левее существенного спектра.

2. Если $\lambda=\bar{\lambda}$, то оператор $\bar{H}^{\lambda}(\mathbf{0})$ имеет бесконечное число собственных значений, лежащих на отрезке $[-\bar{\rho}, 0] \subset \sigma_{\mathrm{ess}}\left(\bar{H}^{\lambda}(\mathbf{0})\right)$.

3. Если $\lambda=\left(\lambda_{1}, \lambda_{2}, \lambda_{3}\right)$ таково, что $\lambda_{1,2,3} \geqslant \lambda^{*}$, mо оператор $\bar{H}^{\lambda}(\mathbf{0})$ uмеет бесконечное число собственных значений, лежащих в лакуне существенного спектра, точнее, на интервале $(-\rho, 0)$.

Обозначим через $\mathcal{V}$ множество функций, квадратично-интегрируемых на $\mathbb{T}^{3}$ и периодических по каждому аргументу с периодом $\pi$. Ясно, что $\mathcal{V}$ является подпространством пространства $L_{2}\left(\mathbb{T}^{3}\right)$.

ЗАмечание 2. Собственные функции невозмущенного оператора $H(\mathbf{0})$ принадлежат ядру возмущающего оператора $\bar{V}^{\lambda}$ (см. ниже доказательство леммы 3). Класс таких возмущающих операторов является широким. Например, если в качестве $v$ взять функцию из $\mathcal{V}$, ортогональную функции, тождественно равной единице, то можно проверить, что собственные функции невозмущенного оператора $H(\mathbf{0})$ принадлежат ядру соответствующего возмущающего оператора $\bar{V}^{\lambda}$, определенного с помощью $v$. При этом результаты теоремы 2 верны для более общего случая, когда возмущающий оператор $\bar{V}^{\lambda}$ определен с помощью $v \in \mathcal{V}$. 


\section{3. О СПЕКТРЕ ДВУХЧАСТИЧНОГО ОПЕРАТОРА ШРЕДИНГЕРА}

Рассмотрим самосопряженный оператор $\bar{h}_{\alpha}(k), k \in \mathbb{T}^{3}, \alpha=1,2,3$, действующий в $L_{2}\left(\mathbb{T}^{3}\right)$ по формуле $(1)$ :

$$
\bar{h}_{\alpha}(k)=h_{0}^{\alpha}(k)-\mu_{\alpha} \mathbf{v}-\lambda_{\alpha} \overline{\mathbf{v}} ;
$$

здесь $h_{0}^{\alpha}(k)$ - оператор умножения на функцию

$$
\mathcal{E}_{k}^{(\alpha)}(p)=\frac{\varepsilon(p)}{m_{\beta}}+\frac{\varepsilon(k-p)}{m_{\gamma}}
$$

и $\mathbf{v}_{\alpha}:=\mu_{\alpha} \overline{\mathbf{v}}+\lambda_{\alpha} \mathbf{v}-$ интегральный оператор с ядром

$$
v_{\alpha}(p-s)=\frac{\mu_{\alpha}}{8 \pi^{3}}+\lambda_{\alpha} \sum_{i=1}^{3} \cos \left(p_{i}-s_{i}\right) .
$$

Введем оператор $\tilde{h}_{\alpha}(k)$, действующий в $L_{2}\left(\mathbb{T}^{3}\right)$,

$$
\tilde{h}_{\alpha}(k)=\tilde{h}_{0}^{\alpha}(k)-\mathbf{v}_{\alpha},
$$

где $\tilde{h}_{0}^{\alpha}(k)$ - оператор умножения на функцию

$$
\widetilde{E}_{k}^{(\alpha)}(p)=\sum_{i=1}^{3}\left(\frac{1}{m_{\beta}}+\frac{1}{m_{\gamma}}-\sqrt{\frac{1}{m_{\beta}^{2}}+\frac{2}{m_{\beta} m_{\gamma}} \cos 2 k_{i}+\frac{1}{m_{\gamma}^{2}}} \cos 2 p_{i}\right) .
$$

Пусть $u: L_{2}\left(\mathbb{T}^{3}\right) \rightarrow L_{2}\left(\mathbb{T}^{3}\right)$ - унитарный оператор, определенный формулой

$$
(u f)(p)=f\left(p-\frac{\theta(k)}{2}\right)
$$

где

$$
\theta(k)=\left(\theta_{1}\left(k_{1}\right), \theta_{2}\left(k_{2}\right), \theta_{3}\left(k_{3}\right)\right), \quad \theta_{i}\left(k_{i}\right)=\arccos \frac{\frac{1}{m_{\beta}}+\frac{1}{m_{\gamma}} \cos 2 k_{i}}{\sqrt{\frac{1}{m_{\beta}^{2}}+\frac{2}{m_{\beta} m_{\gamma}} \cos 2 k_{i}+\frac{1}{m_{\gamma}^{2}}}} .
$$

Тогда

$$
\left(u^{-1} f\right)(p)=f\left(p+\frac{\theta(k)}{2}\right), \quad f \in L_{2}\left(\mathbb{T}^{3}\right) .
$$

Лемма 5. Оператор $\bar{h}_{\alpha}(k)$ унитарно эквивалентен оператору $\tilde{h}_{\alpha}(k)$ :

$$
\tilde{h}_{\alpha}(k)=u^{-1} \bar{h}_{\alpha}(k) u \text {. }
$$

ДокАЗАТЕЛЬСТво. Поскольку имеет место представление

$$
\mathcal{E}_{k}^{(\alpha)}(p)=\sum_{i=1}^{3}\left[\frac{1}{m_{\beta}}+\frac{1}{m_{\gamma}}-\sqrt{\frac{1}{m_{\beta}^{2}}+\frac{2}{m_{\beta} m_{\gamma}} \cos 2 k_{i}+\frac{1}{m_{\gamma}^{2}}} \cos \left(2 p_{i}-\frac{\theta_{i}\left(k_{i}\right)}{2}\right)\right],
$$

справедливо равенство

$$
\begin{aligned}
& \left(\bar{h}_{0}^{(\alpha)}(k) u f\right)(p)= \\
& \quad=\sum_{i=1}^{3}\left(\frac{1}{m_{\beta}}+\frac{1}{m_{\gamma}}-\sqrt{\frac{1}{m_{\beta}^{2}}+\frac{2}{m_{\beta} m_{\gamma}} \cos 2 k_{i}+\frac{1}{m_{\gamma}^{2}}} \cos \left(2 p_{i}-\frac{\theta_{i}\left(k_{i}\right)}{2}\right)\right) f\left(p-\frac{\theta(k)}{2}\right) .
\end{aligned}
$$


Отсюда легко получаем, что

$$
\left(u^{-1} \bar{h}_{0}^{\alpha}(k) u f\right)(p)=\sum_{i=1}^{3}\left(\frac{1}{m_{\beta}}+\frac{1}{m_{\gamma}}-\sqrt{\frac{1}{m_{\beta}^{2}}+\frac{2}{m_{\beta} m_{\gamma}} \cos 2 k_{i}+\frac{1}{m_{\gamma}^{2}}} \cos \left(2 p_{i}\right)\right) f(p),
$$

т. е. $u^{-1} h_{0}^{\alpha}(k) u=\tilde{h}_{0}^{\alpha}(k)$. Ясно, что

$$
\begin{aligned}
\left(u^{-1} \mathbf{v}_{\alpha} u f\right)(p) & =u^{-1}\left(\int_{\mathbb{T}^{3}} v_{\alpha}(s-p) f\left(s-\frac{\theta(k)}{2}\right) d s\right)= \\
& =\int_{\mathbb{T}^{3}} v_{\alpha}\left(s-\left(p+\frac{\theta(k)}{2}\right)\right) f\left(s-\frac{\theta(k)}{2}\right) d s .
\end{aligned}
$$

В последнем интеграле, сделав замену $s-\theta(k) / 2=t$, имеем

$$
\left(u^{-1} \mathbf{v}_{\alpha} u f\right)(p)=\int_{\mathbb{T}^{3}} v_{\alpha}(t-p) f(t) d t
$$

таким образом, $u^{-1} \mathbf{v}_{\alpha} u=\mathbf{v}_{\alpha}$. Лемма доказана.

Лемма 6. Пусть либо $m_{\beta} \neq m_{\gamma} u k \in \mathbb{T}^{3}$, либо $m=m_{\beta}=m_{\gamma} u\left|k_{i}\right| \neq \pi / 2$ для всех $i=1,2,3$. Тогда интеграл

$$
\int_{\mathbb{T}^{3}} \frac{\varphi(s) d s}{\widetilde{E}_{k}^{(\alpha)}(s)-m^{(\alpha)}(k)}
$$

сходится для всех $\varphi \in C\left(\mathbb{T}^{3}\right)$.

ДокАЗАТЕЛЬСТво. В обоих случаях, сформулированных в условии леммы,

$$
\frac{1}{m_{\beta}^{2}}+\frac{2}{m_{\beta} m_{\gamma}} \cos 2 k_{i}+\frac{1}{m_{\gamma}^{2}}=\left(\frac{1}{m_{\beta}}-\frac{1}{m_{\gamma}}\right)^{2}+\frac{2}{m_{\beta} m_{\gamma}}\left(1+\cos 2 k_{i}\right) \neq 0 .
$$

Поэтому функция $\widetilde{E}_{k}^{(\alpha)}(\cdot)$ достигает своего минимального значения $m^{(\alpha)}(k)$ в точках $p \in \Pi$, где П - множество векторов $k=\left(k_{1}, k_{2}, k_{3}\right) \in \mathbb{T}^{3}$ таких, что координаты вектора $k$ принимают значения 0 или $\pi$. При этом точки минимума являются невырожденными. Так как точки минимума функции $\widetilde{E}_{k}^{(\alpha)}(\cdot)$ невырожденные и этих точек восемь, интеграл (4) существует для всех $\varphi \in C\left(\mathbb{T}^{3}\right)$. Лемма доказана.

ЗАМЕЧАНИЕ 3 . Если $m=m_{\beta}=m_{\gamma}$ и $\left|k_{i}\right|=\pi / 2$ для каждого $i=1,2,3$, то

$$
\widetilde{E}_{k}^{(\alpha)}(s)-m^{(\alpha)}(k)=0 \quad \text { для всех } \quad k \in \mathbb{T}^{3} .
$$

В этом случае, если $\varphi(s) \neq 0$ и $\varphi \in C\left(\mathbb{T}^{3}\right)$, то

$$
\int_{\mathbb{T}^{3}} \frac{|\varphi(s)| d s}{\widetilde{E}_{k}^{(\alpha)}(s)-m^{(\alpha)}(k)} \rightarrow+\infty \quad \text { при } \quad\left(\left|k_{1}\right|,\left|k_{2}\right|,\left|k_{3}\right|\right) \rightarrow(\pi / 2, \pi / 2, \pi / 2) .
$$

Поэтому в силу неравенства $m^{(\alpha)}(k) \geqslant 0$ для всех $k \in \mathbb{T}^{3}$ имеют место оценки

$$
\left(\min _{k \in \mathbb{T}^{3}} \int_{\mathbb{T}^{3}} \frac{|\varphi(s)| d s}{\widetilde{E}_{k}^{(\alpha)}(s)}\right)^{-1}<\infty, \quad\left(\max _{k \in \mathbb{T}^{3}} \int_{\mathbb{T}^{3}} \frac{|\varphi(s)| d s}{\widetilde{E}_{k}^{(\alpha)}(s)}\right)^{-1}<\infty .
$$


Введем обозначение

$$
\Delta_{\alpha}(k ; z)=\prod_{i=0}^{6} \Delta_{\alpha}^{(i)}(k ; z)
$$

где

$$
\begin{aligned}
& \Delta_{\alpha}^{(0)}(k ; z)=1-\frac{\mu_{\alpha}}{8 \pi^{3}} \int_{\mathbb{T}^{3}} \frac{d s}{\widetilde{E}_{k}^{(\alpha)}(s)-z}, \\
& \Delta_{\alpha}^{(i)}(k ; z)=1-\lambda_{\alpha} \int_{\mathbb{T}^{3}} \frac{\cos ^{2} s_{i} d s}{\widetilde{E}_{k}^{(\alpha)}(s)-z}, \quad i=1,2,3, \\
& \Delta_{\alpha}^{(i)}(k ; z)=1-\lambda_{\alpha} \int_{\mathbb{T}^{3}} \frac{\sin ^{2} s_{i-3} d s}{\widetilde{E}_{k}^{(\alpha)}(s)-z}, \quad i=4,5,6 .
\end{aligned}
$$

ЛЕмма 7. Число $z<m^{(\alpha)}(k)$ является собственным значением оператора $\tilde{h}^{(\alpha)}(k)$ тогда и только тогда, когда

$$
\Delta_{\alpha}(k ; z)=0 .
$$

ДокАЗАтЕЛЬСтво. Пусть $z<m^{(\alpha)}(k)$ - собственное значение оператора $\tilde{h}^{(\alpha)}(k)$ и $f$ - соответствующий собственный вектор, т. е. уравнение

$$
\left(\tilde{h}_{\alpha}(k) f\right)(p)=\widetilde{E}_{k}^{(\alpha)}(p) f(p)-\frac{\mu_{\alpha}}{8 \pi^{3}} \int_{\mathbb{T}^{3}} f(s) d s-\lambda_{\alpha} \sum_{i=1}^{3} \int_{\mathbb{T}^{3}} \cos \left(p_{i}-s_{i}\right) f(s) d s
$$

имеет нетривиальное решение $f$. Поскольку $z<m^{(\alpha)}(k)$, то $\widetilde{E}_{k}^{(\alpha)}(p)-z>0$ для всех $p \in \mathbb{T}^{3}$. Поэтому из (5) имеем

$$
f(p)=\frac{1}{\widetilde{E}_{k}^{(\alpha)}(p)-z}\left[\frac{\mu_{\alpha}}{8 \pi^{3}} \int_{\mathbb{T}^{3}} f(s) d s-\lambda_{\alpha} \sum_{i=1}^{3} \int_{\mathbb{T}^{3}} \cos \left(p_{i}-s_{i}\right) f(s) d s\right] .
$$

Отсюда

$$
f(p)=\frac{1}{\widetilde{E}_{k}^{(\alpha)}(p)-z}\left[\mu_{\alpha} c_{0}+\lambda_{\alpha} \sum_{i=1}^{3}\left(c_{i} \cos p_{i}+r_{i} \sin p_{i}\right)\right]
$$

где

$$
c_{0}=\frac{\mu_{\alpha}}{8 \pi^{3}} \int_{\mathbb{T}^{3}} f(s) d s, \quad c_{i}=\int_{\mathbb{T}^{3}} \cos s_{i} f(s) d s, \quad r_{i}=\int_{\mathbb{T}^{3}} \sin s_{i} f(s) d s .
$$

Подставив выражение (6) в (7), получим

$$
\begin{aligned}
c_{0} & =\frac{\mu_{\alpha}}{8 \pi^{3}} \int_{\mathbb{T}^{3}} \frac{1}{\widetilde{E}_{k}^{(\alpha)}(s)-z}\left[\mu_{\alpha} c_{0}+\lambda_{\alpha} \sum_{i=1}^{3}\left(c_{i} \cos s_{i}+r_{i} \sin s_{i}\right)\right] d s, \\
c_{i} & =\int_{\mathbb{T}^{3}} \frac{\cos s_{i}}{\widetilde{E}_{k}^{(\alpha)}(s)-z}\left[\mu_{\alpha} c_{0}+\lambda_{\alpha} \sum_{i=1}^{3}\left(c_{i} \cos s_{i}+r_{i} \sin s_{i}\right)\right] d s, \\
r_{i} & =\int_{\mathbb{T}^{3}} \frac{\sin s_{i}}{\widetilde{E}_{k}^{(\alpha)}(s)-z}\left[\mu_{\alpha} c_{0}+\lambda_{\alpha} \sum_{i=1}^{3}\left(c_{i} \cos s_{i}+r_{i} \sin s_{i}\right)\right] d s .
\end{aligned}
$$


В силу четности функции $\widetilde{E}_{k}^{(\alpha)}(\cdot)$ для всех $i, j, \gamma=1,2,3, i \neq j$ имеют место формулы

$$
\int_{\mathbb{T}^{3}} \frac{\sin s_{i} \sin s_{j} d s}{\widetilde{E}_{k}^{(\alpha)}(s)-z}=\int_{\mathbb{T}^{3}} \frac{\sin s_{i} \cos s_{\gamma} d s}{\widetilde{E}_{k}^{(\alpha)}(s)-z}=0 .
$$

Равенства

$$
\int_{\mathbb{T}^{3}} \frac{\cos s_{i} d s}{\widetilde{E}_{k}^{(\alpha)}(s)-z}=0, \quad \int_{\mathbb{T}^{3}} \frac{\cos s_{i} \cos s_{j} d s}{\widetilde{E}_{k}^{(\alpha)}(s)-z}=0,
$$

также справедливые для всех $i, j, \gamma=1,2,3, i \neq j$, следуют из периодичности функции $\widetilde{E}_{k}^{(\alpha)}(\cdot)$ по каждой переменной с периодом $\pi$ и получаются с помощью замены переменных $t_{i}=s_{i}+\pi$ в интегралах левых частей равенств (10).

С учетом соотношений (9), (10) система уравнений (8) приобретает вид

$$
\begin{aligned}
& c_{0}=\frac{\mu_{\alpha}}{8 \pi^{3}} \int_{\mathbb{T}^{3}} \frac{d s}{\widetilde{E}_{k}^{(\alpha)}(s)-z} c_{0}, \\
& c_{i}=\lambda_{\alpha} \int_{\mathbb{T}^{3}} \frac{\cos ^{2} s_{i} d s}{\widetilde{E}_{k}^{(\alpha)}(s)-z} c_{i}, \\
& r_{i}=\lambda_{\alpha} \int_{\mathbb{T}^{3}} \frac{\sin ^{2} s_{i} d s}{\widetilde{E}_{k}^{(\alpha)}(s)-z} r_{i} .
\end{aligned}
$$

Известно, что линейная система (11) имеет ненулевое решение $\left(c_{0}, c_{1}, c_{2}, c_{3}, r_{1}, r_{2}, r_{3}\right)$ тогда и только тогда, когда $\Delta_{\alpha}(k ; z)=0$.

Обратно, если $\Delta_{\alpha}(k ; z)=0$, то хотя бы одно уравнение из системы (11) имеет ненулевое решение $c_{l}$ или $r_{\alpha}$. Легко проверить, что функция $f(\cdot)$, определенная по формуле $(6)$, является собственной функцией оператора $\tilde{h}_{\alpha}(k)$, соответствующей собственному значению $z<m^{(\alpha)}(k)$. Лемма доказана.

Лемма 8. Справедливы следующие утверждения.

1. Существует число $\lambda_{\alpha}^{0}>0$ такое, что для всех $\lambda_{\alpha} \leqslant \lambda_{\alpha}^{0} u k \in \mathbb{T}^{3}$ имеет место неравенство $\Delta_{\alpha}(k ; z)>0$ для каждого $z<0$.

2. Существуют числа $\bar{\lambda}_{\alpha}^{0}$ и $\bar{\rho}=\bar{\rho}\left(\bar{\lambda}_{\alpha}^{0}\right)$ такие, что для каждого $z \in[-\bar{\rho}, 0)$ имеет место равенство $\Delta_{\alpha}(k ; z)=0$ при $\lambda_{\alpha}=\bar{\lambda}_{\alpha}^{0}$ и для некоторого $k=k(z) \in \mathbb{T}^{3}$.

3. Для любого $\rho>0$ существует число $\lambda_{\alpha}^{*}=\lambda_{\alpha}^{*}(\rho)$ такое, что для всех $\lambda_{\alpha}>\lambda_{\alpha}^{*}$ $u k \in \mathbb{T}^{3}$ имеет место неравенство $\Delta_{\alpha}(k ; z) \neq 0$ для каждого $z \in[-\rho, 0)$.

ДоказАтельство. Отметим, что из условия $\mu_{\alpha} \leqslant \mu_{\alpha}^{0}$ вытекает, что $\Delta_{\alpha}^{(0)}(k ; z)>0$ для всех $k \in \mathbb{T}^{3}$ и $z<0$.

1. Положим (см. замечание 2)

$$
\lambda_{\alpha}^{0}=\left(\max _{k, i} \int_{\mathbb{T}^{3}} \frac{\cos ^{2} s_{i} d s}{\widetilde{E}_{k}^{(\alpha)}(s)}\right)^{-1} .
$$

Пусть $\lambda_{\alpha} \leqslant \lambda_{\alpha}^{0}$. Тогда для всех $z<0$ имеем

$$
\Delta_{\alpha}^{(i)}(k ; z)=1-\lambda_{\alpha} \int_{\mathbb{T}^{3}} \frac{\cos ^{2} s_{i} d s}{\widetilde{E}_{k}^{(\alpha)}(s)-z} d s>1-\lambda_{\alpha}^{0} \max _{k, i} \int_{\mathbb{T}^{3}} \frac{\cos ^{2} s_{i} d s}{\widetilde{E}_{k}^{(\alpha)}(s)}=0 .
$$


Отсюда и из неравенства [15]

$$
\int_{\mathbb{T}^{3}} \frac{\cos ^{2} s_{i} d s}{\widetilde{E}_{k}^{(\alpha)}(s)-z} \geqslant \int_{\mathbb{T}^{3}} \frac{\sin ^{2} s_{i} d s}{\widetilde{E}_{k}^{(\alpha)}(s)-z} d s
$$

следует, что $\Delta_{\alpha}^{(3+i)}(k ; z) \geqslant \Delta_{\alpha}^{(i)}(k ; z)>0$ для всех $i=1,2,3$ и $z<0$, любых $\lambda_{\alpha} \leqslant \lambda_{\alpha}^{0}$ и $k \in \mathbb{T}^{3}$. Следовательно, $\Delta_{\alpha}(k ; z)>0$ для всех $z<0, \lambda_{\alpha} \leqslant \lambda_{\alpha}^{0}$ и $k \in \mathbb{T}^{3}$.

2. Положим (см. замечание 2)

$$
\begin{aligned}
& \lambda_{\alpha}^{*}(i)=\left(\min _{k} \int_{\mathbb{T}^{3}} \frac{\cos ^{2} s_{i} d s}{\widetilde{E}_{k}^{(\alpha)}(s)}\right)^{-1}=\left(\int_{\mathbb{T}^{3}} \frac{\cos ^{2} s_{i} d s}{\widetilde{E}_{k^{*}}^{(\alpha)}(s)}\right)^{-1}, \\
& \lambda_{\alpha}^{0}(i)=\left(\max _{k} \int_{\mathbb{T}^{3}} \frac{\cos ^{2} s_{i} d s}{\widetilde{E}_{k}^{(\alpha)}(s)}\right)^{-1}=\left(\int_{\mathbb{T}^{3}} \frac{\cos ^{2} s_{i} d s}{\widetilde{E}_{k^{0}}^{(\alpha)}(s)}\right)^{-1} .
\end{aligned}
$$

Пусть $\lambda_{\alpha}(i) \in\left[\lambda_{\alpha}^{0}(i), \lambda_{\alpha}^{*}(i)\right]$. Тогда

$$
\Delta_{\alpha}^{(i)}\left(k^{0} ; 0\right)=1-\lambda_{\alpha}(i) \int_{\mathbb{T}^{3}} \frac{\cos ^{2} s_{i} d s}{\widetilde{E}_{k^{0}}^{(\alpha)}(s)} \leqslant 1-\lambda_{\alpha}^{0}(i) \int_{\mathbb{T}^{3}} \frac{\cos ^{2} s_{i} d s}{\widetilde{E}_{k^{0}}^{(\alpha)}(s)}=0 .
$$

Поскольку $\lim _{z \rightarrow-\infty} \Delta_{\alpha}^{(i)}\left(k^{0} ; 0\right)=1$, в силу непрерывности функции $\Delta_{\alpha}^{(i)}\left(k^{0} ; \cdot\right)$ существует $z_{0} \in(-\infty, 0)$ такое, что $\Delta_{\alpha}^{(i)}\left(k^{0} ; z_{0}\right)=0$. Положим $\bar{\rho}=-z_{0}$. Пусть $z \in(-\bar{\rho}, 0)$. Тогда

$$
\begin{aligned}
& \Delta_{\alpha}^{(i)}\left(k^{0} ; z\right)=1-\lambda_{\alpha}(i) \int_{\mathbb{T}^{3}} \frac{\cos ^{2} s_{i} d s}{\widetilde{E}_{k^{0}}^{(\alpha)}(s)-z}<1-\lambda_{\alpha}(i) \int_{\mathbb{T}^{3}} \frac{\cos ^{2} s_{i} d s}{\widetilde{E}_{k^{0}}^{(\alpha)}(s)+\bar{\rho}}=\Delta_{\alpha}^{(i)}\left(k^{0} ;-\bar{\rho}\right)=0, \\
& \Delta_{\alpha}^{(i)}\left(k^{*} ; z\right)=1-\lambda_{\alpha}(i) \int_{\mathbb{T}^{3}} \frac{\cos ^{2} s_{i} d s}{\widetilde{E}_{k^{*}}^{(\alpha)}(s)-z}>1-\lambda_{\alpha}^{*}(i) \int_{\mathbb{T}^{3}} \frac{\cos ^{2} s_{i} d s}{\widetilde{E}_{k^{*}}^{(\alpha)}(s)}=0 .
\end{aligned}
$$

Поэтому в силу непрерывности функции $\Delta_{\alpha}^{(i)}(\cdot ; z)$ существует $k \in \mathbb{T}^{3}$ такое, что $\Delta_{\alpha}^{(i)}(k ; z)=0$. Отсюда для выполнения утверждения 2 достаточно взять $\bar{\lambda}_{\alpha}^{0}=\lambda_{\alpha}(i)$ для одного $i \in\{1,2,3\}$.

3. Положим

$$
\lambda_{\alpha}^{*}=\left(\min _{k, i} \int_{\mathbb{T}^{3}} \frac{\sin ^{2} s_{i} d s}{\widetilde{E}_{k}^{(\alpha)}(s)-\rho}\right)^{-1} .
$$

Пусть $\lambda_{\alpha}>\lambda_{\alpha}^{*}$. Тогда из (12) получим

$$
\begin{aligned}
\Delta_{\alpha}^{(i)}(k ; \rho) \leqslant \Delta_{\alpha}^{(3+i)}(k ; \rho) & =1-\lambda_{\alpha} \int_{\mathbb{T}^{3}} \frac{\sin ^{2} s_{i} d s}{\widetilde{E}_{k}^{(\alpha)}(s)-\rho}< \\
& <1-\lambda_{\alpha}^{*} \min _{k, i} \int_{\mathbb{T}^{3}} \frac{\sin ^{2} s_{i} d s}{\widetilde{E}_{k}^{(\alpha)}(s)-\rho}=0 .
\end{aligned}
$$

Поэтому в силу монотонного убывания функции $\Delta_{\alpha}^{(i)}(k ; \cdot)$ имеет место неравенство $\Delta_{\alpha}^{(i)}(k ; z) \leqslant \Delta_{\alpha}^{(3+i)}(k ; z)<0$ для всех $z \in[-\rho, 0)$ и $i=1,2,3$. Отсюда следует утверждение 3 . 
Теперь докажем леммы 4 и 3 из раздела 2.

ДокАЗАТЕЛьство леммы 4. Докажем каждое из трех утверждений леммы.

1. Пусть $\lambda=\left(\lambda_{1}, \lambda_{2}, \lambda_{3}\right)$ таков, что

$$
\lambda_{i} \leqslant \lambda^{0}=\min _{\alpha \in\{1,2,3\}} \lambda_{\alpha}^{0}, \quad i=1,2,3 .
$$

Тогда согласно утверждению 1 леммы 8 для всех $z<0$ и $k \in \mathbb{T}^{3}$ имеет место неравенство $\Delta_{\alpha}(k ; z)>0$ при каждом $\alpha=1,2,3$. Отсюда с учетом леммы 7 каждый из операторов $\tilde{h}_{\alpha}(k), \alpha=1,2,3$, не имеет отрицательных собственных значений для всех $k \in \mathbb{T}^{3}$. Поэтому из равенства (3) получаем, что $\sigma\left(\bar{H}_{\alpha}^{\lambda}(\mathbf{0})\right)=\left[0, M_{\mathbf{0}}\right]$ для всех $\alpha=1,2,3$. В силу леммы 1 отсюда имеем первое утверждение леммы 4.

2. Положим $\bar{\lambda}=\left(\bar{\lambda}_{1}^{0}, \lambda_{2}, \lambda_{3}\right)$, где $\bar{\lambda}_{1}^{0}-$ число, найденное в лемме 8 . Тогда согласно утверждению 3 леммы 8 , принимая во внимание лемму 7 , получаем, что для каждого $z \in[-\bar{\rho}, 0)$ существует $k=k(\bar{\rho}) \in \mathbb{T}^{3}$ такое, что число $z$ является собственным значением оператора $\tilde{h}_{1}(k)$. Согласно равенству $(3)$ имеем $[-\bar{\rho}, 0) \subset \sigma\left(\bar{H}_{1}^{\lambda}(\mathbf{0})\right)$. Отсюда и из леммы 1 получаем второе утвержение леммы 4.

3. Пусть $\lambda^{*}=\max _{\alpha \in\{1,2,3\}} \lambda_{\alpha}^{*}$ и $\lambda^{*}=\lambda^{*}(\rho)$ - число, найденное в лемме 8 , а $\rho$ соответствующее положительное число. Пусть $\lambda=\left(\lambda_{1}, \lambda_{2}, \lambda_{3}\right)$ таков, что $\lambda_{i}>\lambda^{*}$ для всех $i=1,2,3$. Тогда согласно утверждению 2 леммы 8 при всех $z \in[-\rho, 0)$ и $k \in \mathbb{T}^{3}$ имеет место неравенство $\Delta_{\alpha}(k ; z) \neq 0$ для каждого $\alpha=1,2,3$. Следовательно, по лемме 7 оператор $\tilde{h}_{\alpha}(k)$ не имеет собственных значений на отрезке $[-\rho, 0)$. Поэтому из равенства (3) получаем соотношение

$$
\sigma\left(\bar{H}^{\lambda}(\mathbf{0})\right) \cap(-\rho, 0)=\varnothing .
$$

Для всех $\lambda_{\alpha}>\lambda^{*}$ и $k \in \mathbb{T}^{3}$ имеет место неравенство $\Delta_{\alpha}(k ; z)<0$ при каждом $z \in[-\rho, 0)$. Отсюда, с учетом того что $\lim _{z \rightarrow-\infty} \Delta_{\alpha}^{(i)}(k ; 0)=1$, в силу непрерывности функции $\Delta_{\alpha}^{(i)}(k ; \cdot)$ существует $z=z(k)<-\rho$ такое, что $\Delta_{\alpha}^{(i)}(k ; z)=0$ для всех $\lambda_{\alpha}>\lambda^{*}$ и $k \in \mathbb{T}^{3}$. Следовательно, по лемме 7 оператор $\tilde{h}_{\alpha}(k)$ имеет собственные значения на полуоси $(-\infty,-\rho)$ для всех $\lambda_{\alpha}>\lambda^{*}$ и $k \in \mathbb{T}^{3}$. Отсюда и из равенства (3) вытекает, что

$$
\sigma\left(\bar{H}_{\alpha}^{\lambda}(\mathbf{0})\right) \cap(-\infty,-\rho) \neq \varnothing .
$$

Следовательно, с учетом леммы 1 мы получаем

$$
\sigma\left(\bar{H}^{\lambda}(\mathbf{0})\right) \cap(-\infty,-\rho) \neq \varnothing
$$

Лемма 4 полностью доказана.

ДокАЗАТЕЛЬство ЛЕммы 3. Для доказательства достаточно показать, что каждая собственная функция $f$ оператора $H(\mathbf{0})$, соответствующая собственному числу, лежащему левее существенного спектра, принадлежит ядру оператора $V^{\lambda}$, т. е. $V^{\lambda} f=0$.

Пусть $z<0$ - собственное значение оператора $H(\mathbf{0})$ и $f$ - соответствующий собственный вектор, т. е. уравнение

$$
H(\mathbf{0}) f=z f
$$


имеет нетривиальное решение $f$. Поскольку $z<0$, то $E_{\mathbf{0}}(p, q)-z>0$ для всех $p, q \in \mathbb{T}^{3}$. Поэтому из (13) имеем

$$
\begin{aligned}
& f(p, q)= \\
& \quad=\frac{1}{E_{\mathbf{0}}(p, q)-z}\left[\frac{\mu_{1}}{8 \pi^{3}} \int_{\mathbb{T}^{3}} f(s, q) d s+\frac{\mu_{2}}{8 \pi^{3}} \int_{\mathbb{T}^{3}} f(p, s) d s+\frac{\mu_{3}}{8 \pi^{3}} \int_{\mathbb{T}^{3}} f(s, p+q-s) d s\right] .
\end{aligned}
$$

Отсюда

$$
f(p, q)=\frac{1}{E_{\mathbf{0}}(p, q)-z}\left[\varphi_{1}(q)+\varphi_{2}(p)+\varphi_{3}(p+q)\right],
$$

где

$$
\begin{aligned}
\varphi_{1}(q) & =\frac{\mu_{1}}{8 \pi^{3}} \int_{\mathbb{T}^{3}} f(s, q) d s, \\
\varphi_{2}(p) & =\frac{\mu_{2}}{8 \pi^{3}} \int_{\mathbb{T}^{3}} f(p, s) d s, \\
\varphi_{3}(p+q) & =\frac{\mu_{3}}{8 \pi^{3}} \int_{\mathbb{T}^{3}} f(s, p+q-s) d s .
\end{aligned}
$$

Подставляя (14) в (15), получим

$$
\begin{aligned}
\varphi_{1}(q) & =\frac{\mu_{1}}{8 \pi^{3}} \int_{\mathbb{T}^{3}} \frac{1}{E_{\mathbf{0}}(s, q)-z}\left[\varphi_{1}(q)+\varphi_{2}(s)+\varphi_{3}(s+q)\right] d s, \\
\varphi_{2}(p) & =\frac{\mu_{2}}{8 \pi^{3}} \int_{\mathbb{T}^{3}} \frac{1}{E_{\mathbf{0}}(p, s)-z}\left[\varphi_{1}(s)+\varphi_{2}(p)+\varphi_{3}(p+s)\right] d s, \\
\varphi_{3}(p+q) & =\frac{\mu_{3}}{8 \pi^{3}} \int_{\mathbb{T}^{3}} \frac{1}{E_{\mathbf{0}}(s, p+q-s)-z}\left[\varphi_{1}(p+q-s)+\varphi_{2}(s)+\varphi_{3}(p+q)\right] d s
\end{aligned}
$$

или, эквивалентно,

$$
\begin{gathered}
\varphi_{1}(q) \Delta_{1}(q ; z)=\frac{\mu_{1}}{8 \pi^{3}} \int_{\mathbb{T}^{3}} \frac{\varphi_{2}(s) d s}{E_{\mathbf{0}}(s, q)-z}+\frac{\mu_{1}}{8 \pi^{3}} \int_{\mathbb{T}^{3}} \frac{\varphi_{3}(s+q) d s}{E_{\mathbf{0}}(s, q)-z}, \\
\varphi_{2}(p) \Delta_{2}(p ; z)=\frac{\mu_{2}}{8 \pi^{3}} \int_{\mathbb{T}^{3}} \frac{\varphi_{1}(s) d s}{E_{\mathbf{0}}(p, s)-z}+\frac{\mu_{2}}{8 \pi^{3}} \int_{\mathbb{T}^{3}} \frac{\varphi_{3}(p+s) d s}{E_{\mathbf{0}}(p, s)-z}, \\
\varphi_{3}(p+q) \Delta_{3}(p+q ; z)=\frac{\mu_{3}}{8 \pi^{3}} \int_{\mathbb{T}^{3}} \frac{\varphi_{2}(s) d s}{E_{\mathbf{0}}(s, p+q-s)-z}+\frac{\mu_{3}}{8 \pi^{3}} \int_{\mathbb{T}^{3}} \frac{\varphi_{1}(p+q-s) d s}{E_{\mathbf{0}}(s, p+q-s)-z},
\end{gathered}
$$

где

$$
\begin{aligned}
\Delta_{1}(q ; z) & =1-\frac{\mu_{1}}{8 \pi^{3}} \int_{\mathbb{T}^{3}} \frac{d s}{E_{\mathbf{0}}(s, q)-z}, \\
\Delta_{2}(p ; z) & =1-\frac{\mu_{2}}{8 \pi^{3}} \int_{\mathbb{T}^{3}} \frac{d s}{E_{\mathbf{0}}(p, s)-z}, \\
\Delta_{3}(p+q ; z) & =1-\frac{\mu_{3}}{8 \pi^{3}} \int_{\mathbb{T}^{3}} \frac{d s}{E_{\mathbf{0}}(s, p+q-s)-z} .
\end{aligned}
$$

В последних интегралах правых частей уравнений системы (16), совершая замены переменных $s+p=t, s+q=t p+q-s=t$, с учетом периодичности подынтегральных 
функций и неравенств $\Delta_{\alpha}(p ; z)>0$, справедливых при $z<0$ и $\mu_{\alpha} \leqslant \mu_{\alpha}^{0}$, имеем

$$
\begin{aligned}
\varphi_{1}(q) & =\frac{\mu_{1}}{8 \pi^{3}} \frac{1}{\Delta_{1}(q ; z)} \int_{\mathbb{T}^{3}} \frac{\varphi_{2}(s) d s}{E_{\mathbf{0}}(s, q)-z}+\frac{\mu_{1}}{8 \pi^{3}} \int_{\mathbb{T}^{3}} \frac{\varphi_{3}(s) d s}{E_{\mathbf{0}}(s-q, q)-z}, \\
\varphi_{2}(p) & =\frac{\mu_{2}}{8 \pi^{3}} \frac{1}{\Delta_{2}(p ; z)} \int_{\mathbb{T}^{3}} \frac{\varphi_{1}(s) d s}{E_{\mathbf{0}}(p, s)-z}+\frac{\mu_{2}}{8 \pi^{3}} \int_{\mathbb{T}^{3}} \frac{\varphi_{3}(s) d s}{E_{\mathbf{0}}(p, s-p)-z}, \\
\varphi_{3}(p+q) & =\frac{\mu_{3}}{8 \pi^{3}} \frac{1}{\Delta_{3}(p+q ; z)} \int_{\mathbb{T}^{3}} \frac{\varphi_{2}(s) d s}{E_{\mathbf{0}}(s, p+q-s)-z}+\frac{\mu_{3}}{8 \pi^{3}} \int_{\mathbb{T}^{3}} \frac{\varphi_{1}(s) d s}{E_{\mathbf{0}}(p+q-s, s)-z} .
\end{aligned}
$$

Поскольку $E_{\mathbf{0}}(\cdot, \cdot)$ является функцией, периодической по каждому аргументу с периодом $\pi$, такой же является и функция $\Delta_{\alpha}(\cdot ; z)$ для каждого $\alpha=1,2,3$. Поэтому каждая из функций $\varphi_{\alpha}(\cdot), \alpha=1,2,3$, определенных на $\mathbb{T}^{3}$, является периодической функцией по каждому аргументу с периодом $\pi$. Таким образом, из (14) вытекает, что собственная функция $f$ оператора $H(\mathbf{0})$ является функцией, периодической по каждому аргументу с периодом $\pi$.

Покажем, что $\left(\bar{V}_{\alpha} f\right)(p, q)=0$ для каждого $\alpha=1,2,3$. Запишем $\bar{V}_{1} f$ в виде

$$
\left(\bar{V}_{1} f\right)(p, q)=\sum_{i=1}^{3} \cos p_{i} \int_{\mathbb{T}^{3}} \cos s_{i} f(s, p) d s+\sum_{i=1}^{3} \sin p_{i} \int_{\mathbb{T}^{3}} \sin s_{i} f(s, p) d s
$$

Совершая здесь замену переменной $s_{i}=t_{i}+\pi$, имеем $\bar{V}_{1} f=-\bar{V}_{1} f$, т. е. $\bar{V}_{1} f=0$.

Совершенно аналогично показывается, что $\bar{V}_{2} f=\bar{V}_{3} f=0$.

\section{4. АСИМПТОТИКА ДЛЯ ЧИСЛА СОБСТВЕННЫХ ЗНАЧЕНИЙ ОПЕРАТОРА $H(0)$}

Пусть $\left.U: L_{2}\left(\left(\mathbb{T}^{3}\right)^{2}\right) \rightarrow L_{2}\left((2 \mathbb{T})^{3}\right)^{2}\right)$ - унитарный оператор, определенный по формуле

$$
(U f)(p, q)=8 f(2 p, 2 q)
$$

где $2 \mathbb{T}:=(-2 \pi, 2 \pi]$. Тогда

$$
\left(U^{-1} f\right)(p, q)=\frac{1}{8} f\left(\frac{p}{2}, \frac{q}{2}\right)
$$

Обозначим через $\bar{H}$ самосопряженный оператор, действующий в гильбертовом пространстве $\left.L_{2}\left((2 \mathbb{T})^{3}\right)^{2}\right)$ по формуле

$$
\bar{H}=\bar{H}_{0}-\mu_{1} \widetilde{V}_{1}+\mu_{2} \widetilde{V}_{2}+\mu_{3} \widetilde{V}_{3},
$$

где $\bar{H}_{0}$ - оператор умножения на функцию

$$
E_{\mathbf{0}}\left(\frac{p}{2}, \frac{q}{2}\right)=\frac{1}{m_{1}} \varepsilon\left(\frac{p}{2}\right)+\frac{1}{m_{2}} \varepsilon\left(\frac{q}{2}\right)+\frac{1}{m_{3}} \varepsilon\left(\frac{p+q}{2}\right),
$$

4 Теоретическая и математическая физика, т. 182, № 3, 2015 г. 
а операторы $\widetilde{V}, \alpha=1,2,3$, заданы формулами

$$
\begin{aligned}
& \left(\widetilde{V}_{1} f\right)(p, q)=\frac{1}{(2 \pi)^{3}} \int_{\mathbb{T}^{3}} f(p, 2 s) d s \\
& \left.\left(\widetilde{V}_{2} f\right)(p, q)=\frac{1}{(2 \pi)^{3}} \int_{\mathbb{T}^{3}} f(2 s, q) d s, \quad f \in L_{2}\left((2 \mathbb{T})^{3}\right)^{2}\right), \\
& \left(\widetilde{V}_{3} f\right)(p, q)=\int_{\mathbb{T}^{3}} f(2 s, p+q-2 s) d s .
\end{aligned}
$$

Легко доказывается следующая

Лемма 9. Оператор $H(\mathbf{0})$ унитарно эквивалентен оператору $\bar{H}$ :

$$
\bar{H}=U^{-1} H(\mathbf{0}) U \text {. }
$$

ЛЕмма 10. Каждая собственная функиия $f \in L_{2}\left(\left((2 \mathbb{T})^{3}\right)^{2}\right)$ оператора $\bar{H}$, соответствующая собственному значению $z<0$, является периодической функцией по каждому аргументу с периодом $2 \pi$.

ДокАзАтЕЛЬство. Пользуясь периодичностью функции $E_{\mathbf{0}}(\cdot, \cdot)$ по каждому аргументу с периодом $\pi$, рассуждая аналогично доказательству леммы 3 , получим утверждение леммы.

Обозначим через $H$ самосопряженный оператор, действующий в гильбертовом пространстве $L_{2}\left(\left(\mathbb{T}^{3}\right)^{2}\right)$ по формуле

$$
H=H_{0}-\mu_{1} V_{1}+\mu_{2} V_{2}+\mu_{3} V_{3},
$$

где $H_{0}$ - оператор умножения на функцию (17).

ЛЕмма 11. Каждое собственное значение оператора $H(\mathbf{0})$, лежащее левее существенного спектра, является собственным значением оператора $H$ с той же кратностью, и обратно.

ДокАЗАтельство. Пусть $\left.f \in L_{2}\left((2 \mathbb{T})^{3}\right)^{2}\right)$ - собственная функция оператора $\bar{H}$, соответствующая собственному значению $z<0$. Тогда по лемме 10 функция $f$ является периодической каждому аргументу с периодом $2 \pi$. Отсюда имеем

$$
\int_{\mathbb{T}^{3}} f(p, 2 s) d s=\frac{1}{8} \int_{(2 \mathbb{T})^{3}} f(p, t) d t=\int_{\mathbb{T}^{3}} f(p, t) d t,
$$

другими словами,

$$
\left(\widetilde{V}_{1} f\right)(p, q)=\frac{1}{(2 \pi)^{3}} \int_{\mathbb{T}^{3}} f(p, s) d s .
$$

Проводя аналогичные вычисления, получим

$$
\begin{aligned}
& \left(\widetilde{V}_{2} f\right)(p, q)=\frac{1}{(2 \pi)^{3}} \int_{\mathbb{T}^{3}} f(s, q) d s, \\
& \left(\widetilde{V}_{3} f\right)(p, q)=\int_{\mathbb{T}^{3}} f(s, p+q-s) d s,
\end{aligned}
$$


Эти представления показывают, что функция $\left.g \in L_{2}\left((\mathbb{T})^{3}\right)^{2}\right)$, заданная как сужение функции $\left.f \in L_{2}\left((2 \mathbb{T})^{3}\right)^{2}\right)$ на $\left((\mathbb{T})^{3}\right)^{2}$, является собственной функцией оператора $H$, а число $z$ - соответствующим собственным числом.

Обратно, если $\left.g \in L_{2}\left((\mathbb{T})^{3}\right)^{2}\right)$ - собственная функция оператора $H$, соответствующая собственному значению $z<0$, то функция, периодическая по каждому аргументу с периодом $2 \pi$, заданная как $\left.g \in L_{2}\left((2 \mathbb{T})^{3}\right)^{2}\right)$, является собственной функцией оператора $\bar{H}$, а число $z$ - соответствующим собственным значением. При этом каждое собственное значение оператора $\bar{H}$, лежащее левее существенного спектра, является собственным значением оператора $H$ с той же кратностью, и обратно. Отсюда и из леммы 9 получим доказательство леммы 3.

Простое вычисление показывает, что

$$
\int_{\mathbb{T}^{3}} \frac{d s}{\varepsilon(s)}=\int_{\mathbb{T}^{3}} \frac{d s}{\varepsilon(s / 2)} .
$$

Поэтому

$$
\mu_{\alpha}^{0}=8 \pi^{3} \frac{m_{\beta}+m_{\gamma}}{m_{\beta} m_{\gamma}}\left(\int_{\mathbb{T}^{3}} \frac{d s}{\varepsilon(s / 2)}\right)^{-1} .
$$

Следующая теорема доказана в работе [8].

ТЕОрема 3. Пусть $\mu_{\alpha}=\mu_{\alpha}^{0}, \mu_{\beta}=\mu_{\beta}^{0} u \mu_{\gamma} \leqslant \mu_{\gamma}^{0}$ при $\alpha \neq \beta \neq \gamma$. Тогда существует предел

$$
\lim _{z \rightarrow-0}\left(-\frac{1}{\ln (-z)} \bar{N}(z)\right)=\mathcal{U}_{0}, \quad \mathcal{U}_{0}>0,
$$

где $\bar{N}(z)$ - число собственнъх значений оператора $H$ (с учетом кратности), лежащих левее $z<0$.

Доказательство теоремы 1 вытекает из леммы 11 и теоремы 3.

Заметим, что положительное число $\mathcal{U}_{0}$ есть число, найденное в работе [4], которое зависит от числа виртуальных уровней двухчастичных подгамильтонианов и от отношений масс частиц.

Благодарности. Авторы выражают благодарность рецензенту за чтение работы и полезные замечания.

\section{Список литературы}

[1] В. Н. Ефимов, ЯФ, 12:5 (1970), 1080-1091.

[2] R. D. Amado, J. V. Noble, Phys. Rev. D, 5:8 (1972), 1992-2002.

[3] С. П. Меркурьев, Л. Д. Фаддеев, Квантовая теория рассеяния для систем нескольких частии, Наука, М., 1985.

[4] A. V. Sobolev, Commun. Math. Phys., 156:1 (1993), 101-126.

[5] H. Tamura, Nagoya Math. J., 130 (1993), 55-83.

[6] С. Н. Лакаев, З.Э. Муминов, Функи. анализ и его прил., 37:3 (2003), 80-84.

[7] Ж. И. Абдуллаев, С. Н. Лакаев, ТМФ, 136:2 (2003), 231-245.

[8] S. Albeverio, S. N. Lakaev, Z. I. Muminov, Ann. Henri Poincaré, 5:4 (2004), 743-772.

[9] С. Н. Лакаев, М.Э. Муминов, ТМФ, 135:3 (2003), 478-503. 
[10] Г. М. Жислин, Тр. ММО, 9, 1960, 81-120.

[11] М. Э. Муминов, ТМФ, 159:2 (2009), 299-317.

[12] М.Э. Муминов, ТМФ, 164:1 (2010), 46-61.

[13] S. Albeverio, S. N. Lakaev, Z. I. Muminov, Math. Nachr., 280:7 (2007), 699-716.

[14] М. Рид, Б. Саймон, Методы современной математической физики, т. 4: Анализ операторов, Мир, М., 1982.

[15] М.Э. Муминов, А. М. Хуррамов, ТМФ, 177:3 (2013), 482-496.

Поступила в редакцию 4.07.2014, после доработки 4.09.2014 